\title{
System efficient integration of standby control and heat pump storage systems in manufacturing processes
}

\author{
Florian Schlosser $^{\mathrm{a}, *}, \S$, Jan-Peter Seevers ${ }^{\mathrm{a}}{ }^{\S}$, Ron-Hendrik Peesel ${ }^{\mathrm{a}}$, Timothy Gordon \\ Walmsley \\ aUniversity of Kassel, Dep. Sustainable Products and Processes, Kurt-Wolters-Straße 3, 34125 Kassel, Germany \\ ${ }^{b}$ Sustainable Process Integration Laboratory - SPIL, NETME Centre, Faculty of Mechanical Engineering, Brno \\ University of Technology - VUT Brno, Technická 2896/2, 61669 Brno, Czech Republic \\ *corresponding author: schlosser@upp-kassel.de \\ $\S$ : Both authors contributed equally to this work.
}

\begin{abstract}
Prerequisite for system efficiency towards an industrial energy transition is the reducing of energy demand on the process level. In typical manufacturing systems with machine tools and washing machines, the proper design of intelligent standby control and heat pump storage system (HPS) represent high efficiency. The integration of HPS is complicated due to high non-continuity, especially when implementing a standby control system. Our approach aims at designing one single HPS for multiple heat sources and sinks. Robust design should consider the various influencing material flow system factors. For the generation of stochastic heating and cooling demand sum curves, 512 Design of Experiments-based material flow simulations for each of three standby scenarios have been conducted. These curves serve as input data for HPS sizing and dynamic thermal system simulation. The combined integration of an HPS and a practical standby control system offers the best compromise in terms of system efficiency with significantly lower investment costs and only slightly lower energy savings than ideal standby operation. Compared to the initial state, the electrical energy demand of the machines can be reduced by $27 \%$ and both the heating $(83 \%)$ and cooling (48 $\%$ ) demand can be efficiently covered by HPs.
\end{abstract}

Keywords: Design of Experiments, heat pump, total site heat integration, standby control, material flow simulation

\section{Introduction}

As part of the global energy transition to limit climate change, the Paris Agreement envisages a reduction of greenhouse gas (GHG) emissions to $40 \%$ by 2040, starting from the base year 1990 [1]. In 2016 around $21.4 \%$ of Germany's total energy consumption was needed for process heat. Usually, process heat is generated by fossil-fired steam boilers for economic benefits. The share of renewable energies on this consumption was only $5.2 \%$. By 2050, the share of renewable energies in German industry is planned to rise to $63 \%$ [2]. The integration of variable renewable energy requires electrification in all sectors [3] and flexibility in terms of adapting energy demand to supply. Against this background, the energy supply of industrial sites needs to convert from a centralized conventional fossil fuel heat plant to a decentralized electrical based one. Besides renewable energy, higher energy efficiency is an effective way to reduce GHG emissions significantly. According to the onion layer model in Figure 1, energy efficiency is to be systematically increased from the process through technical equipment to the public grid [4]. 


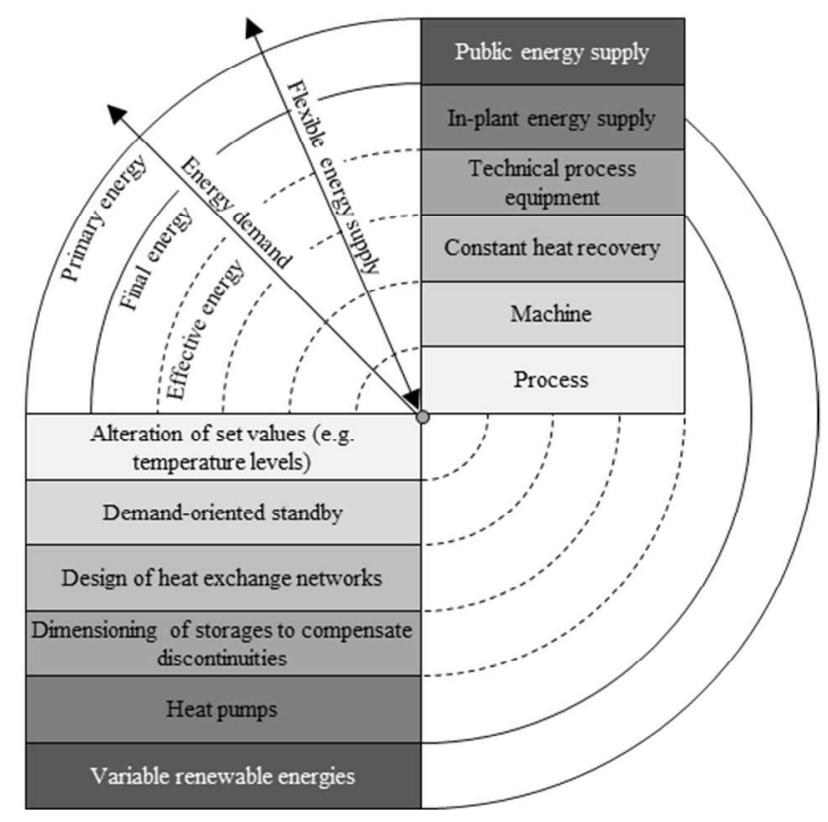

Figure 1. Energy efficiency in manufacturing lines according to the onion layer model

After the optimal dimensioning of the machine components at the process level, the machine level requires the implementation of a demand-oriented standby control. In the following three layers, as much energy as possible is recovered at the line level using Process Integration and Pinch Analysis methods [4]. This implicates heat recovery and the proper dimensioning of storage and heat pumps (HPs).

HPs are electrical-driven heat recovery technologies that upgrade waste heat to a suitable process temperature level to reduce heating and cooling demands. In quasi-continuous processes, HPs can be integrated energy-efficiently across the pinch temperature using pinch analysis based on the Grand Composite Curve (GCC) [5]. In large manufacturing sites, heat recovery (HR) is usually limited due to the non-continuous character of the occurrence of sources and sinks caused by different target outputs of manufacturing lines and shut down times for maintenance and repair purposes. In such processes, heating and cooling demands fluctuate with time. HPs combining heating and cooling best run in steady operation points. Heat recovery loops (HRLs) combined with thermal energy storages (TESs) can further increase heat recovery [6].

HPs are also used in manufacturing sites to link heat sinks and sources of different processes. Grüninger and Wellig [7] demonstrated the particular suitability of the integration of HPs in metal processing sites using the shape and pinch temperature of the GCC. While Preuß [8], Hennecke [9] and Hlavica [10] still present a HP recovering the heat from heat sources like laser cutting machines, compressed air systems, bath cooling or the exhaust gases for surface treatment and space heating, this constant heat sources may be reduced or eliminated in future energy supply systems. Moreover, sinks such as space heating are only available during the heating period. Manufacturing lines, in particular, offer the possibility of process integration due to their obligatory sequence of machine tools as a heat source and washing machines as a heat sink for the integration of HPs at the process level. Junge et al. [11] consider one machine tool and one washing machine. In a laboratory environment, they dimensioned a HP which both cools the machine tool and provides heat to the washing machine. However, they made strict assumptions regarding shut down times. Likewise, Watanabe [12] created a simple solution to integrate HPs by working in three operational modes. If heating and cooling tasks coincide, the HP couples one heat source and one heat sink. In the other 
two cases, either the recooling energy is transferred to the hall air (HP in cooling mode) or the hall air is used as a heat source (HP in heating mode). First, this simple technical solution requires high investment cost due to the number of different HPs for every washing machine and second, this can cause additional heating or cooling demand for the production hall.

In contrast to the existing approaches, this study aims to find dimensioning solutions for a heat pump storage system (HPS) that couples all heat sinks and heat sources in a manufacturing system. In this way, the low-temperature waste heat generated at the process level can transfer to heat sinks at the process level. However, the main problem with the correct dimensioning of such a HPS for an entire manufacturing system is that manufacturing machines have a volatile energy demand over time due to fluctuating material throughput and operating conditions [13]. This volatility can even increase with a more energy efficient central standby control [14]. Despite this volatility, Stoltze [15] assumed that the specific storage capacity requirement decreases with an increasing number of discontinuous, fluctuating sinks and sources. To confirm this hypothesis, a Design of Experiments (DoE) based (Figure 2.1) material and energy flow simulation (Figure 2.2) in this study should consider different standby strategies as well as complex material flow dependencies and fluctuating sources of interference. Based on the generated heat and cooling demand stochastic time series (Figure 2.3), a Pinch Analysis based dimensioning of storages and HPs (like in [16]) is carried out (Figure 2.4). Following dimensioning, a step-wise simulation evaluates the dynamic thermal behavior and possible heat recovery rates (Figure 2.5). It is also the goal of this study to conclude whether a HP, a standby strategy, or a combination of both, leads to higher energy efficiency.
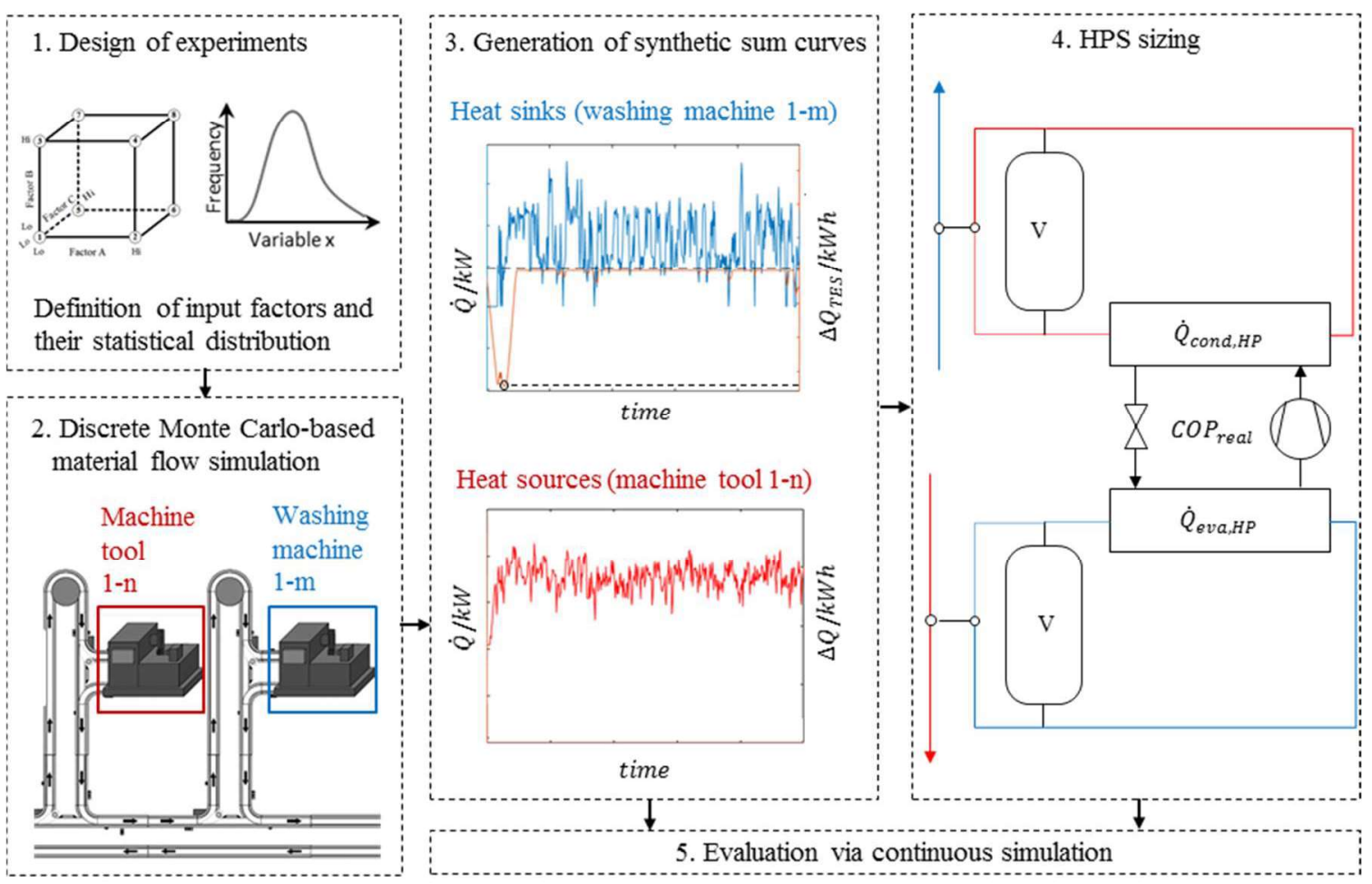

Figure 2. Structure and methodology of the study in five steps 
The structure of this paper is as follows: Section 2 gives an overview of the state of the art regarding the energy efficiency of manufacturing lines. Section 3 describes the methodology to dimension the HPS. This methodology consists of the five methodological steps shown in Figure 2. In Section 4 the methodology is applied to a material flow system in a gear manufacturing line, which consists of several manufacturing machines like cutting, grinding, milling, and honing processes with cooling demand, as well as several cleaning machines with heating demand. A practical approach is presented using graphical techniques based on pinch analysis for HP integration in non-continuous processes. The results and their scalability and transferability of this comprehensive case study are explained in Chapter 5. Finally, Chapter 5 concludes the results of this paper and gives an outlook.

\section{Energy efficient manufacturing lines: an overview}

According to the Onion Layer model (Figure 1), the energy efficiency of manufacturing lines can be increased on the process level by:

(1) proper dimensioning of machine's components like e.g. cooling compressor (Section 2.1.),

(2) reducing the setpoint temperatures of the cleaning machines, on machine level by optimizing the material flow and standby control (2.2.), or

(3) coupling heat sources (machine tools) and sinks (parts washing machines) on manufacturing system/line level (2.3.).

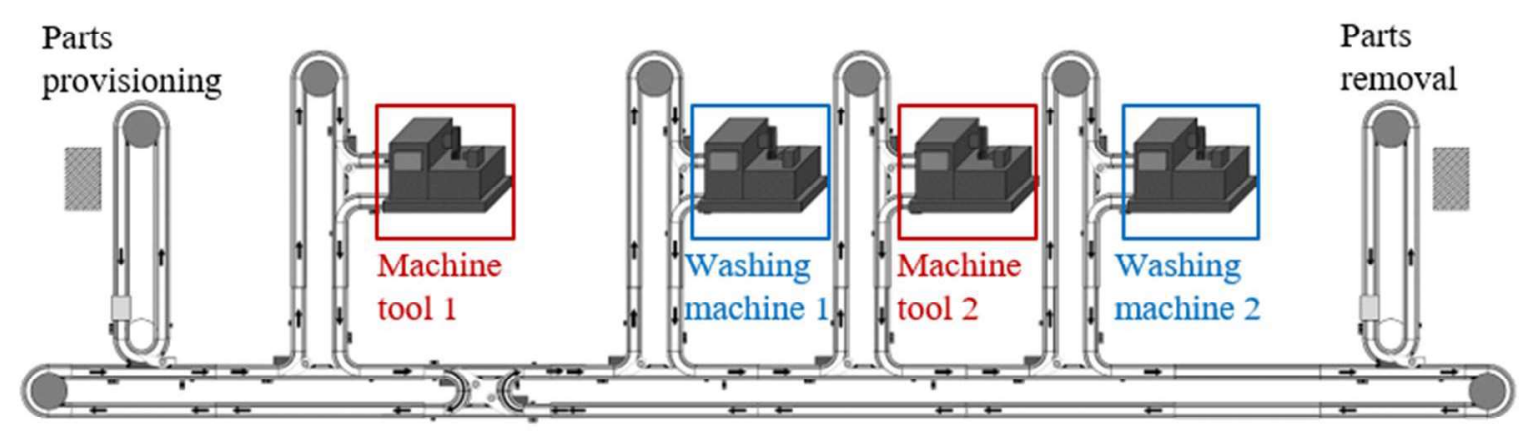

Figure 3. Exemplary manufacturing line consisting of two heat sinks and two heat sources

\subsection{Machine tools and washing machines}

Parts washing machines are necessary, for example, to wash off cooling lubricants, oils or other organic impurities resulting from contact with the parts during the processing of their surfaces. The washing medium is water. The washing temperature has a considerable influence on the cleaning process. Equipment cleaning requires a water temperature of around $50-60^{\circ} \mathrm{C}$. In the final cleaning stage, the washed components are rinsed off with warm water in a separate section of the system.

Cleaning technology is a key differentiation for washing machines [17]. A distinction must be made between two types of process control. On the one hand, continuous parts cleaning systems wash components using conveyor belts. On the other hand, there are chamber part cleaning systems with which the products are cleaned in batches. In this process, the cleaning steps take place one after the other in the same closed machine area. While the continuous parts cleaning machines have a relatively continuous heat demand, the chamber parts cleaning systems have a non-continuous heat demand due to batch operation [18].

In typical machining operation (e.g. milling, turning, grinding and honing), energy efficiency mostly depends on the energy demand for process conditioning (cooling, lubrication), with the main drives 
(main spindle, feed axes) only accounting for a small proportion compared to the active cooling units [19]. As a result, the potential energy savings are determined by measures to improve the machine periphery. In practice, the refrigerant compressor of the auxiliary chillers usually runs continuously; the excess flow rate is controlled at partial load by a hot gas bypass, which represents the most costeffective solution for partial load control under the given requirements for temperature stability. However, the compressor work required in partial load is entirely dissipated, i.e. dissipated as waste heat to the machine hall. [20].

By implementing a HP in between two processes of the production system, the cooling system becomes independent of the ambient temperature. This ensures the cooling of the machines at any time, and the auxiliary chillers become obsolete while also providing hot water for important washing and cleaning processes.

working
warm-keeping
operational
readiness
stand-by
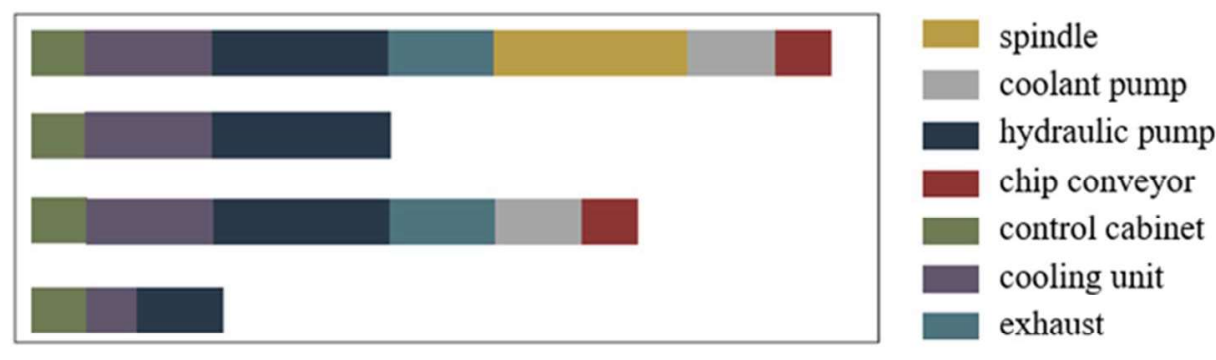

Figure 4. Energy demands of a machine tool at component level (according to [9])

\subsection{Material flow and standby control}

However, HPS couple heat sources and sinks, needing a continuous operation, to an extent, of both sides to some extension [16]. Continuous operation can be affected by fluctuating target outputs and by planned and unplanned production interruptions. Production interruptions occur at weekends, during scheduled shifts changeovers and breaks. In addition, there are also unexpected interruptions in the regular production process. In the planning phase of a production system, due to missing or changing information, an optimal design of the production system can only be approximately achieved [21]. For example, the adaptation of the component geometry or the use of another material can lead to shifts in cycle times and production times. Particularly in the mass production of components with highly coordinated process steps, low machine utilization due to careful capacity planning, economy-related fluctuations in production volume, bottleneck operations, human behavior, processing time fluctuations, pauses and unforeseeable errors can all lead to interruptions in the production flow and thus to unwanted machine downtimes.

Production fluctuations significantly affect production costs and energy efficiency, especially when the base load energy demand is relatively high and independent of the production load or several parts produced. This is because a sizeable proportion of the base load is accounted for by operational readiness of production systems and by the necessary supply technology, such as compressed air systems, machine cooling, cooling lubricant supply, room air conditioning and lighting. Depending on the usage profile and study, the energy demand in waiting or partial load operation accounts for $50-80 \%$ of the annual energy demand of a machine [22]. Machines or components that are ready for operation should often not be switched off due to the risk of losing production reliability. With intelligent, central standby control systems, the systems always receive the current forecast arrival time of the next part to be processed. Using this information, the systems can switch to the optimal energy state and shut down 1-n components, depending on the predicted duration of the processing pause [14]. 
Figure 4 shows an example of a machine tool with four operational states. The different lengths of the horizontal columns illustrate the distinct levels of the average electrical power demand in the respective operational states and the respective machine components. Depending on how many components can be switched off or throttled in a standby mode, the power demand can be significantly reduced from the operational state "waiting for part" or also called "operational readiness". Several standby modes can exist. This depends on the switch-off options specified by the machine manufacturer. The implementation of standby control strategies increases energy efficiency and reduces production cost significantly. However, standby strategies also increase the level of noncontinuity of the process which can be a problem for waste heat recovery and HPS integration. If, for example, all heat sinks shut down and all heat sources are still operating at some point of operation time, the storage or HP will need to be oversized compared to a constant load case.

\section{Methods}

The approach of this paper consists of three key components. The first component defines and selects relevant factors influencing the fluctuation of the cumulative cooling and heating demand curves of the productions system. The second component comprises the DoE and Monte Carlo based material flow simulation, which generates the stochastic sum profiles of cumulative cooling and heating demands. Lastly, the third component considers HPS dimensioning based on dynamic thermal simulation runs using the stochastic demand profiles as an input.

\subsection{Definition of criteria}

To dimension a robust HP, it is vital to consider as many input factors as possible together with their stochastic distributions. This study identifies seven main factors. In Table 1 these factors are divided into two groups. The first group affects the maximum and minimum power value of the production system's cumulative cooling and heating demand profiles. The second group also influences the minimum, maximum and average energy demands of the system. Its primary influence, however, is on the variance of the difference between total cooling and heating demands.

Without a standby strategy in the manufacturing system, the cooling and heating demands fluctuate relatively little because the power demands do not vary greatly in the various operational states. In contrast, standby strategies increase the variance of the difference between cooling and heating demand, depending on the fluctuation frequency and the reduction in power. In general, part buffers increase the variations as the processes in a manufacturing line become more independent of each other. If different production lines or different fixed machining operations are considered as part of the HPS system network, these material flow-related independencies increase the variations as well. Process fluctuation due to, for example, wear can also influence the level of variance. If the machine and line utilization is $100 \%$ or $0 \%$, the variance of the difference between cooling and heating demand is 0 . Other utilization rates increase the variance. Unplanned production interruptions in the manufacturing system cause higher variances in power differences. Production interruptions always affect both machines at the same time [14]. This is a hard restriction and contradicts material flow optimization approaches in real running production systems. To increase the overall productivity of a production line, parts storages are usually integrated in the material flow to avoid the interdependence between different machines [21]. Production interruptions can be considered with the parameters mean time before failure (MTBF), mean time to repair (MTTR) and the machine availability (A). These parameters are generally derived from the analysis and measurements of a technical design and calculated as shown in Equation 1. With the help of these parameters and a specific statistical distribution function to be assumed, apparently random production interruptions can be taken into account relatively well. 


$$
A=M T B F /(M T B F+M T T R)
$$

Table 1. Input factor groups affecting the cooling and heating power demand

Input factor group 1 mainly affecting the maximum and minimum of total cooling and heating power demands
Input factor group 2 mainly affecting the variance of the difference between total cooling and heating power demand

If the two input factor groups in Table 1 are combined, a simulation algorithm is needed to provide information about the actual power curves and their differences at each time step. This focus on the time aspect is necessary for two reasons. On the one hand, the total thermal power curves are needed to calculate the average cooling and heating power demand, which can then form the basis for dimensioning the HP. On the other hand, the integrals of the power difference curve between cooling and heating demand can be calculated and used as a basis for dimensioning the storage tanks. In this context, a discrete event-based material flow simulation can obtain detailed information about the temporal sequence of the machine-operational-state-driven power curves.

\subsection{DoE and Monte Carlo-based material flow simulation}

The input values for the material flow simulation show statistical distributions of their possible values. A prediction of each statistical distribution is only possible with a degree of uncertainty. If the input values and their expected distribution are available with sufficient certainty, the Monte Carlo (MC) method can be applied. In a MC simulation, numerous simulation runs are undertaken. In $\mathrm{MC}$, each simulation run randomly selects a value from each input factor distribution. Due to the determined statistical distribution of the input values, some values are selected more frequently than others. These probability-based simulations then lead to a statistical distribution of the output variables. The analysis of variance can help estimate the minimum number of simulation runs. In general, as many simulations as possible should be performed to ensure independence from the number of runs. The law of large numbers is regarded as the primary justification for the MC method. If detailed statistical distributions of all input factors are not available or if the number and variety of input factors are very high, DoE is used to determine the influence of the variation of input parameters on measurable quantities relevant for evaluation. With a certain number of independent variables, the use of factorial experiments, instead of the one-factor-at-a-time method, is recommended. Factorial experiments are efficient in assessing the effects on the quality and possible interactions of several factors.

The material flow simulation used in this paper, which is fed with the influencing factors described in Section 3.1, applies modular design. Depending on the size of the system (input factor 1), the various modules can be easily connected. The remaining input factors 2-8 and their value ranges or statistic distributions can be specified via input fields and look-up tables. In Figure 5, the seven existing material flow simulation modules are shown. Module 3 can be used both as a machine tool 
(heat source) and as a parts washing machine (heat sink). Both the operational states and the energy demands can be defined for the respective machine.

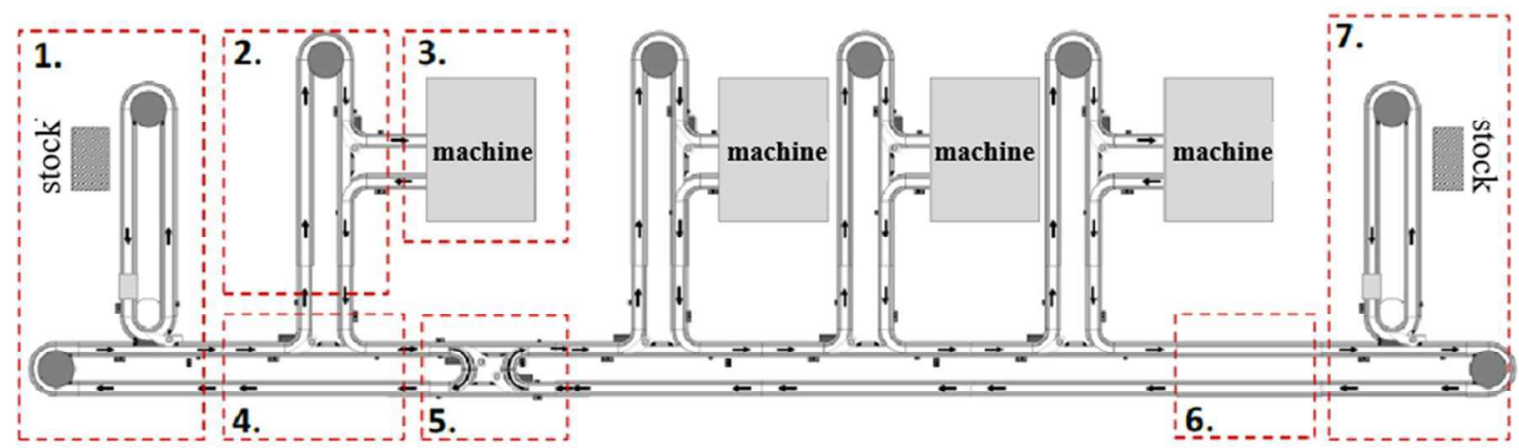

Figure 5. Material flow simulation modules: Parts provisioning (1), parts buffer (2), machining station (3), conveyor belt modules (4)-(6) and parts removal (7); according to [14].

During the simulation run, a table is created showing the current operational status and energy demands (e.g. cooling or heating) for each time step. At the end of the simulation run, the sum curve of all cooling power demands and the sum curve of all heating power demands are calculated. These two sum profiles then serve as input for the HPS dimensioning, as described in the following section 3.3. Figure 6 gives an overview of the methodology with the three central components, including an exemplary representation of the sum curves of cooling and heating demand.

\subsection{Dimensioning of Heat Pump Storage system}

Due to non-continuous operating machines in a material flow system, existing approaches aim at creating a framework to simplify the coupling of machine tools and washing machines. The challenge of HP integration in non-continuous processes can use a mathematical programming approach [23]. Becker et al. [24] present a mixed integer linear programming method to simultaneously target the heat recovery and the integration of energy conversion systems like HPs and other utilities in multiperiod multi-time problems. Improved cost solutions are achievable by mixed integer non-linear programming superstructure synthesis method for industrial HPs [25]. Such methods are usually complex in terms of input data needed and computing time. Stampfli et al. [26] introduced a simple graphical approach for batch processes using Coefficient of Performance (COP) curves based on Time Slice Model (TSM) for choosing the optimal evaporation temperature depending on the condensation temperature and emitted heat. The sizing of the HP and TES can be done by the supply and demand curves of the Time Pinch Analysis [27]. With respect to the variance and probability of the heating and cooling demand, in terms of duration, time of occurrence and height, the suitable dimensioning of storages and HPs must be determined. The GCC and the Indirect Sink and Source Profile (ISSP) method guarantee the thermodynamically correct dimensioning of HPs and storages for a typical stream-wise-repeating period (SROP) of one week. A constant operation of the HP for different manufacturing scenarios must be ensured. Because of the strong discontinuity without the formation of time slices in recurring batches in this study, the methodology is based on the Time Average Model (TAM) and uses the GCC and sink and source curves for the energy targeting as well as HP, TES and HRL sizing according to Schlosser et al [28]. The TAM uses average values across all operating time slices. This approach assumes that the heat storage is always available so that all batch processes can take place at any time and in any order. Based on the generated sum curve of all heat sinks and sources according to Figure 2, the HP and storage (Figure 6.3) can now be dimensioned. This procedure is shown in more detail in Figure 6. 


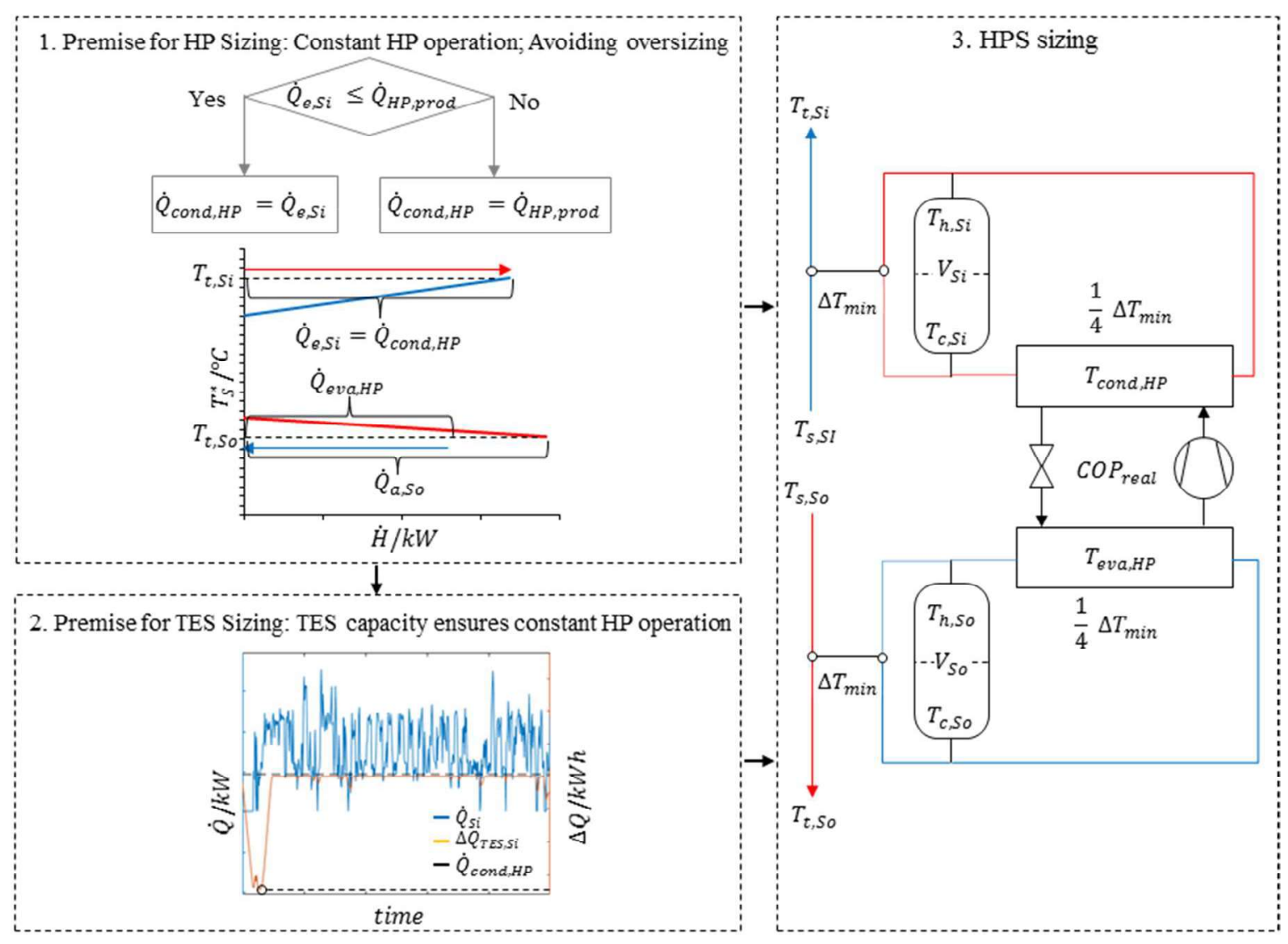

Figure 6. HPS sizing (3.): Dimensioning of the HP by GCC (1.) and sizing of the source and sink-side ST by demand and supply Curve (2.)

The first step is to analyze the availability of heat sinks and source for the integration of a HP. Figure 6.1 displays the minimal average load $\dot{Q}$ of all sink and source streams. This guarantees a constant HP operation and avoids oversizing of equipment. Depending on the shape and gradient of the GCC several HP integration possibilities are conceivable. Often the target temperature of the sink process $T_{t, S i}$ is decisive. Secondly, it must be check whether the heat demand can be fully covered by pumping the existing heat source capacity to the target sink temperature. For the condition $\dot{Q}_{e, S i} \leq \dot{Q}_{H P, p r o d}$, the condensation capacity of the HP is determined according to Equation (5). Otherwise, the emitable heating capacity $\dot{Q}_{e, S i}$ depends on the absorbable heat source $\dot{Q}_{a, S o}$ and $\dot{Q}_{\text {cond }, H P}$ is calculated by Equation (4).

$$
\begin{gathered}
\dot{Q}_{\text {cond }, H P}=\min \left(\dot{Q}_{e, S i} ; \dot{Q}_{H P, p r o d}\right) \\
\operatorname{COP}_{\text {real }}=\zeta_{H P} \cdot \operatorname{COP}{ }_{\text {Carnot }}=\zeta_{H P} \cdot \frac{T_{\text {cond }}}{T_{\text {cond }}-T_{e v}} \\
\dot{Q}_{H P, \text { prod }}=\frac{\sum_{t=1}^{t_{S R O P}} \dot{Q}(t)_{a, S o} \cdot d t}{t_{S R O P} \cdot\left(1-\frac{1}{C O P_{\text {real }}}\right)} \\
\dot{Q}_{e, S i}=\frac{\sum_{t=1}^{t_{S R O P}} \dot{Q}(t)_{e, S i} \cdot d t}{t_{S R O P}}
\end{gathered}
$$


The corresponding evaporation capacity $\dot{Q}_{e v a, H P}$ accounts for a real COP (quality grade $\zeta_{H P}=0.55$ [29]) according to the Equation (3). Condensation $T_{c o n d, H P}$ and evaporation $T_{\text {eva,HP }}$ temperatures are defined by Equations (7) and (8) according to the GCC Figure 6.1 whereby two heat transfers are considered (one between process stream and HRL and another one between HP and HRL). In Addition, $4 / 4$ of the minimum temperature difference $\Delta \mathrm{T}_{\text {min }}$ must be ensured for the heat exchanger (HEX) problem between process stream and HRL. For the latent heat transfer from the evaporator or condenser to the HRL, only a stream-specific minimum temperature difference of $3 / 4 \Delta \mathrm{T}_{\min }$ is necessary due to the high heat transfer coefficient. Since the process streams are already shifted by $1 / 2 \cdot \Delta \mathrm{Tmin}$ in the GCC, the total difference of condensation and evaporation temperature is $5 / 4 \cdot \Delta \operatorname{Tmin}$.

$$
\begin{gathered}
\dot{Q}_{e v a, H P}=\dot{Q}_{c o n d, H P}-P_{e l}=\dot{Q}_{c o n d, H P} \cdot\left(1-\frac{1}{C O P_{\text {real }}}\right) \\
T_{\text {cond }, H P}=T_{t, S i}+\frac{5}{4} \cdot \Delta \mathrm{T}_{\text {min }} \\
T_{\text {eva }, H P}=T_{t, S o}-\frac{5}{4} \cdot \Delta \mathrm{T}_{\text {min }}
\end{gathered}
$$

Due to the temporal incongruence between the constant output of the HP and the no-continuous demand of the sink, a TES must be used to bridge the gap. Since the balance of the transferred heat must be equal after a SROP, the capacity $Q_{T E S, i}$ to be buffered is calculated from the maximum difference between demand and HP according to Figure 6.2. The minimum TES capacity depends on the order in which the storage tank is charged and discharged. As shown in Figure 6.2, the algorithm (9) cumulates only negative or positive differences between HP output and demand to determine the maximum capacity $Q_{T E S, \max }$ to be compensated by the TES. Stratified tanks (ST) or Fixed Temperature and Variable Mass (FTVM) storage can be used as TES devices. Due to the half storage volume, a ST is selected as storage. The volume of the TES $V_{T E S}$ is calculated as shown under (9), line 12. If the available heat source or sink is not completely covered by the evaporator or condenser, a hot and cold utility compensation redundant to the HP is installed.

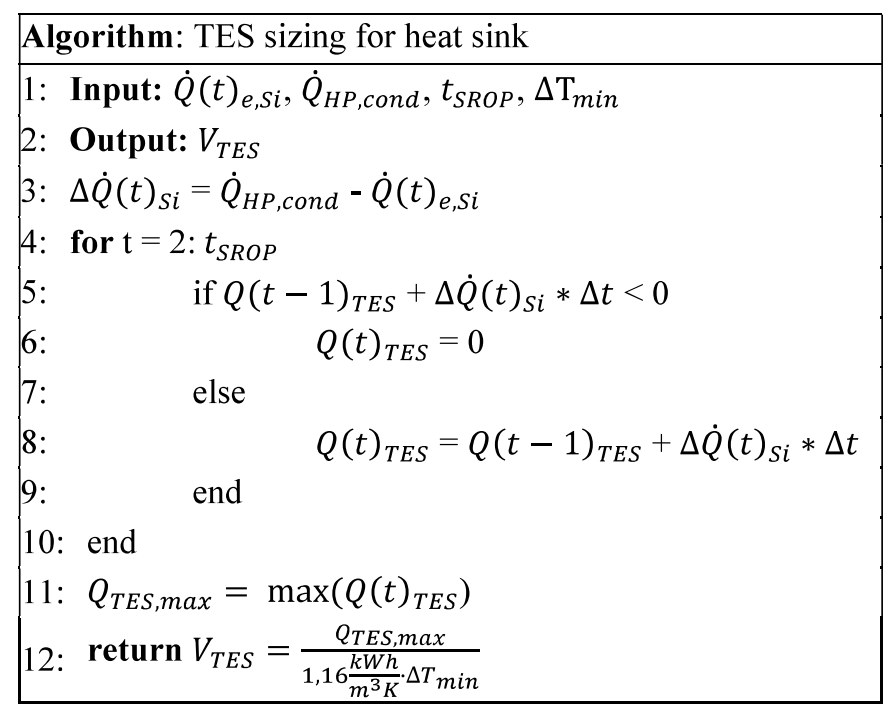




\subsection{Evaluation via continuous simulation studies}

The stochastic time series of the heat sources and sink processes are used as input data for the thermal simulation of the HRL, as depicted in Figure 6. For the sensitive simulation study, a minimal heating demand, as introduced in 3.3, is chosen as the design strategy. The tank size is designed for each standby strategy according to the sink and source curves based on Time Pinch Analysis. The temperatures and loads of the HP and ST are calculated as described in Section 3.3.

The HRL (Figure 6.3) simulation model consists of HEX, ST tank, HP and a two-stage control system. The system control (Figure 7.a) ensures sufficient stratification in the ST and deactivates sinks or sources with a certain hysteresis if threshold values are exceeded. The mass flow control (Figure 7.b) sets the $\mathrm{T}_{\text {hot }}$ and $\mathrm{T}_{\text {cold }}$ in the $\mathrm{ST}$.

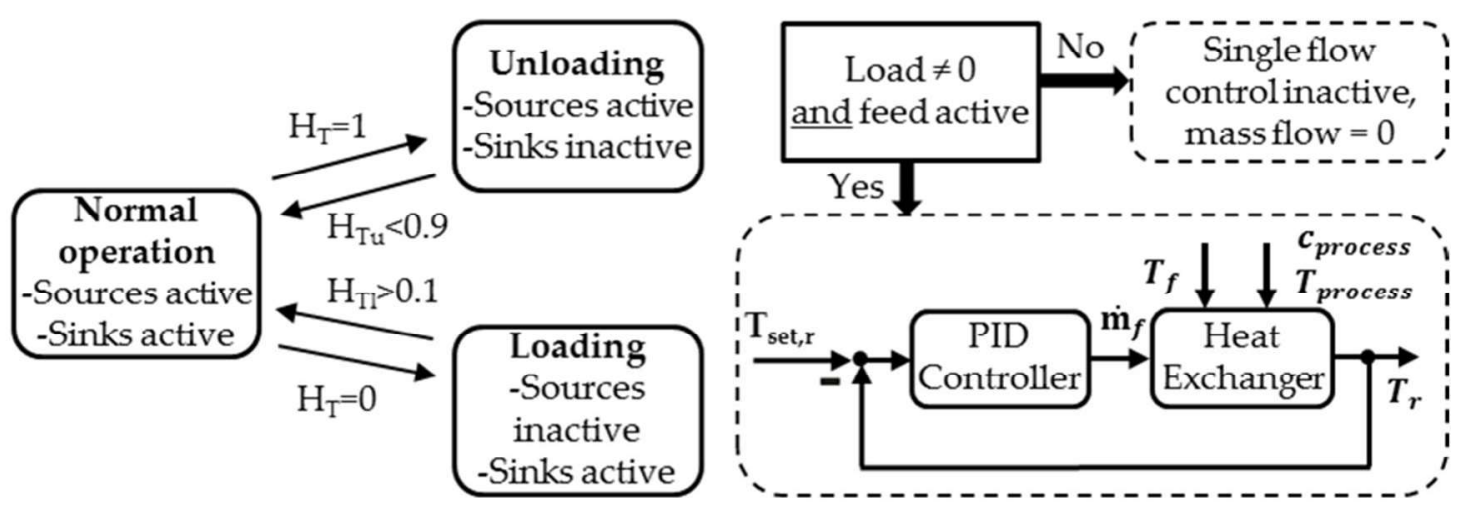

(a)

(b)

Figure 7. System control depending on the thermocline position and (b) single mass flow control adjusting $T_{\text {hot }}$ and $T_{\text {cold }}$

The HEXs are modelled according to the Effectiveness-NTU approach [30]. The VLH model based on the multi-node approach is chosen as the modelling method for the ST [28]. The number of nodes for the ST is determined by the Courant number and inequality.

$$
c=\frac{u \cdot \Delta t}{\Delta x} \leq 1 \Leftrightarrow \Delta t \leq\left|\frac{\rho \cdot V}{4 \cdot \Delta \dot{m}_{\max } \cdot n}\right|
$$

\section{Case study}

The investigated production site is a single part and component manufacturing plant, which produces toothed wheels and shafts for gear manufacturing. Figure 8 shows the corresponding simulation model of the investigated manufacturing line with 15 machines. Machines M1-7, M9, M11 are machine tools with their cooling demands. Machines W8, W10, W12 and W13 are washing machines with heating demands. Machines 14-15 are simple laser marking systems with negligible heating and cooling demands. There are three various kinds of parts machined in this material flow system. The machining sequence for part 1 is: M1-M4-M7-W8, the sequence for part 2 is: M2-M5-M9-W10 and for part 3: M3-M6-M11-W12. Washing machine W13 is stand-by equipment for all three parts that actives when one of the other three washers are occupied. 


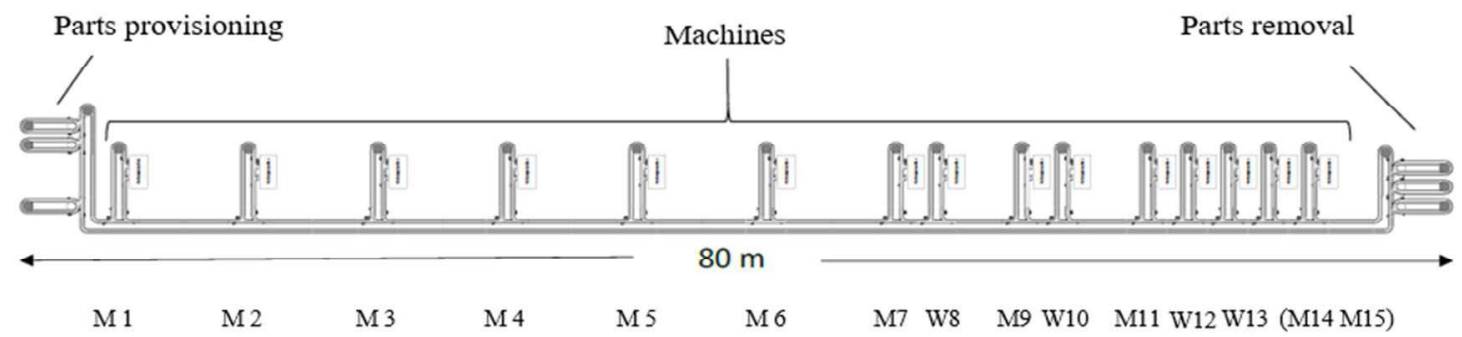

Figure 8. Material flow simulation model of the case study consisting of machine tools $(M 1-7, M 9, M 11, M 14-15)$ and washing machines (W8,W10,W12,W13).

The production comprises several lines with a high degree of automation and networking. The production is designed for a 3-shift operation and 1600-1800 parts per day. However, the production of parts per day can vary widely. Usually, target outputs for one production week are set for every week. The real weekly output of the material flow system though depends on the parts, which are provided to the respective manufacturing line and on the availability of all machining stations, conveyor belts and parts removal at the end of the line.

\subsection{Specification of input factors and DoE}

The input factors number 1-5 (Table 1) describe the influence of system design variations. In this case study, the aim is to investigate the possible integration of a HPS in the existing system. The information regarding the input factors 6-8 is based on historical information. The general process variations in the existing system are relatively weak, difficult to measure and less relevant than input factors 7 and 8 . Therefore, this case study focusses on input factors 7 and 8 .

Two variables represent input factor 7 . The first is the time required to load a free parts carrier in the parts provision area ("loadtime"). The second is the number of carriers that are loaded each with one part at the same point of time ("loadsize"). Both variables have a direct influence on the part throughput of the line. In order to achieve a high target output, both "loading time" and "load size" should be kept as low as possible.

Two variables also represent factor 8 . First is the machine availability A and second is the MTTR (3.1). The simulation requires a statistical distribution of these values. An "Erlang distribution" is used for the MTTR, and a "negative exponential distribution" is used for the MTBF. Given Equation (1), A and MTTR, the MTBF can be calculated.

We define realistic scenarios for the statistical fluctuation over the manufacturing lifetime. In Table 2 the $2^{9}$ - factorial design (512 experiments) is shown with 9 factors each having two levels (low and high). Although it is a classic full factorial $\mathrm{DoE}$, the $\mathrm{MC}$ approach is integrated into the case study via the "Erlang distribution" and "negative exponential distribution" for the machine failures.

Table 2. Factorial design with 9 factors on two levels

\begin{tabular}{llllllllll}
\hline Level & M 1 & M 2 & W 8 & M 9 & W 12 & M 1 & M 9 & \multicolumn{2}{c}{ Parts provision } \\
& A & A & A & A & A & MTTR & MTTR & loadsize & loadtime \\
\hline Low & 0.95 & 0.95 & 0.93 & 0.92 & 0.90 & $5 \mathrm{~min}$ & $10 \mathrm{~min}$ & 5 & $2 \mathrm{~min}$ \\
High & 0.99 & 1.00 & 1.00 & 0.99 & 0.98 & $30 \mathrm{~min}$ & $25 \mathrm{~min}$ & 8 & $10 \mathrm{~min}$
\end{tabular}


Based on real energy monitoring data and manual thermal measurements (already presented in [14] and [16]), different mean values for cooling and heat power demand of each machine can be assumed. As mentioned above, system design factors 1-5 including standby strategies can be considered fixed. According to the onion layer model, the implementation of a standby control system takes priority over the integration of an HPS. However, the variety of possible standby controls is large, as is the possible reduction in the power demands of individual machines, which depends on some technical limitations and circumstances [14]. In practice, the programmatic implementation of energy-saving operating states usually takes place in various forms. Often there is no single standby mode, but a multi-stage reduction of the energy demand in different standby modes. The 512 experiments were each extended by 2 scenarios (in total 1536 experiments) to investigate different types of standby managers: a relatively easily implementable state-of-the-art standby manager and an ideal standby manager. Since we only want to show the general interaction between HPS and Standby Manager at this point, it is sufficient to calculate with average values. For example, ramp-up and ramp-down times, considered in detail in [14], were therefore neglected. Another simplification is that there is no differentiation between operational states like "operational readiness", "blocked", "warm-up" or "in failure". These were condensed to "non-working". Table 3 shows the assumed average cooling and heating power demands for the operational states "working", "not-working" and "standby" for the three scenarios no standby ("no stby"), practical ("stby prac.") and ideal ("stby ideal.") standby control.

Table 3. Average thermal load demands in $\mathrm{kW}$ for heat sources and heat sinks in different operational states

\begin{tabular}{|c|c|c|c|c|c|c|c|c|c|c|c|c|c|}
\hline \multirow{3}{*}{$\begin{array}{l}\text { Operationa } \\
\text { states }\end{array}$} & \multirow{2}{*}{\multicolumn{7}{|c|}{ Heat sources }} & \multirow{3}{*}{$\begin{array}{l}\text { Heat } \\
\text { sink } \\
\text { W 8 }\end{array}$} & \multirow{2}{*}{\multicolumn{2}{|c|}{$\begin{array}{l}\text { Heat Heat } \\
\text { sourcesink }\end{array}$}} & \multirow{3}{*}{$\begin{array}{l}\text { Heat } \\
\text { sourc } \\
\text { M11 }\end{array}$} & \multirow{2}{*}{\multicolumn{2}{|c|}{$\begin{array}{l}\text { Heat sinks } \\
e^{-}\end{array}$}} \\
\hline & & & & & & & & & & & & & \\
\hline & M 1 & M 2 & M 3 & M 4 & M 5 & M 6 & M 7 & & M 9 & W10 & & W12 & W13 \\
\hline working & 8,6 & 8,6 & 8,6 & 13,7 & 13,7 & 13,7 & 7,3 & 17,0 & 7,3 & 17,0 & 7,3 & 17,0 & 17,0 \\
\hline stby & 4,6 & 4,6 & 4,6 & 11,6 & 11,6 & 11,6 & 4,1 & 17,0 & 4,1 & 17,0 & 4,1 & 17,0 & 17,0 \\
\hline tby prac. & 2,0 & 2,0 & 2,0 & 4,0 & 4,0 & 4,0 & 1,5 & 4,0 & 1,5 & 4,0 & 1,5 & 4,0 & 4,0 \\
\hline tby ideal & 0,5 & 0,5 & 0,5 & 0,5 & 0,5 & 0,5 & 0,5 & 1,0 & 0,5 & 1,0 & 0,5 & 1,0 & 1,0 \\
\hline
\end{tabular}

\subsection{Sensitivity simulation study of the HPS}

After dimensioning of the HRL, HP and tank size, the simulation analyses 512 weeks (3.2) for each design scenario with a time increment of one minute. One week, as SROP, is a normal production period in the manufacturing of parts, after which a particular target output has to be fulfilled. Finally, the distribution of heat recovery rates for the different standby scenarios are presented as a result of the simulation study. The HPS system (Figure 9) is designed for minimum heating demand ensuring constant operation over all profiles. 


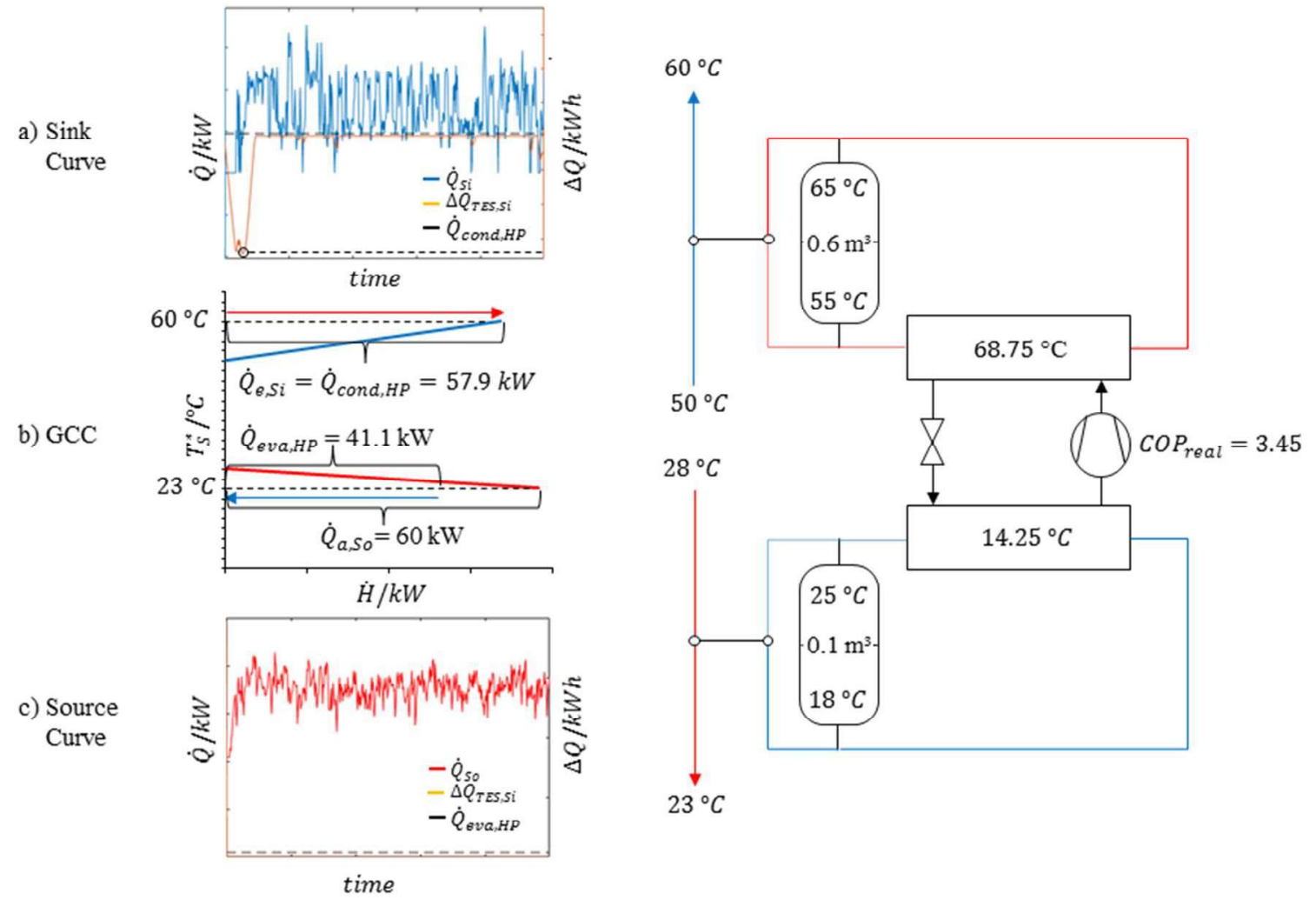

Figure 9. HPS system sized for the initial state without any standby-strategy

Since the target temperatures and the minimum temperature difference remain constant for all scenarios, all scenarios have the same temperature values and COPs. Based on the varying demand profiles of the different scenarios, however, different HPS sizes according to (9) result.

Table 4. Design of HPS system according to 3.3

\begin{tabular}{|c|c|c|c|c|c|}
\hline $\begin{array}{l}\text { ScenarioStandby strategy } \\
\text { Number }\end{array}$ & Design strategy & $C O P_{\text {real }}$ & $\begin{array}{l}\dot{Q}_{\text {cond,HP }} \\
\mathbf{k W}\end{array}$ & $\begin{array}{c}V_{S i, T E S} \\
\mathrm{~m}^{3}\end{array}$ & $\begin{array}{c}V_{\text {So,TES }} \\
\mathrm{m}^{3}\end{array}$ \\
\hline none & & & 57.9 & 0.6 & 0.1 \\
\hline practical & Minimum demand & 3.45 & 24.1 & 2.5 & 0.1 \\
\hline ideal & & & 14.0 & 3.0 & 0.5 \\
\hline
\end{tabular}

The simulation runs were carried out in compliance with the Courant condition for a time step of 1 second. The evaluation criteria of the HPS are the HP coverage rate $\beta$ and the integrated coefficient of performance $\mathrm{COP}_{\beta}$ which also includes the avoided cooling effort.

$$
\beta=\left(\sum_{i=t}^{t_{\text {SROP }}}\left[\frac{\left(\dot{Q}(t)_{\text {cond }, H P}-\dot{Q}(t)_{S i}\right)}{t_{i}}\right]\right)
$$




\section{Discussion of results}

In the following, the distributions of the HP coverage rate for the three scenarios $(3 \times 512)$ are shown in Figure 10. Based on the resulting three heating and cooling demand profile groups, the HPS size (Table 4) varies for the three different scenarios. The HP is designed for the minimum heat demand of all profiles in order to avoid over-dimensioning. The remaining demand is provided by the storage tank and a redundant auxiliary heater. This ensures low capital investment costs.

Frequencies (abs.)

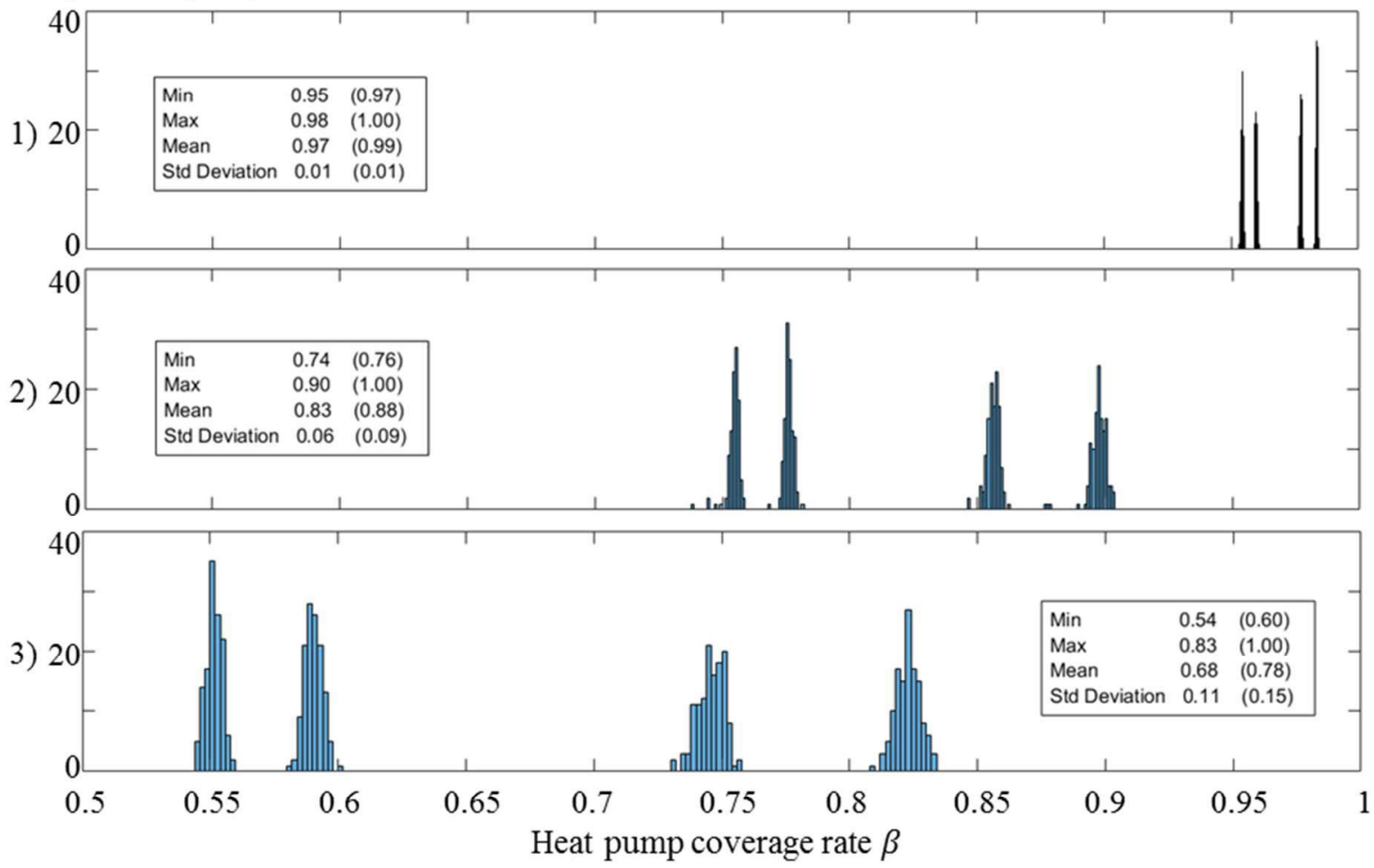

Figure 10. Distribution of the HP coverage rate for the standby strategies 'none' (1), 'practical' (2) and 'ideal' (3) and statistical evaluation of the results with and without thermal losses (in brackets).

The grouping of the frequencies into four clusters results from the DoE of the part provisioning factors load size and load time, which can each have two levels. Since the demand profiles, consisting of various heat sinks and sources, is continuously presented in the scenario (1) without a standby strategy, the HP covers large parts $(97 \%)$ of the heat demand over all clusters in this scenario. For standby strategies, the coverage rates are reduced to $83 \%$ (2) and $68 \%$ (3) due to the increasing fluctuation of demand. If a practical standby control (2) is introduced, this leads to an increase in volatility. The demand profile clusters deviate further out of each other (see increasing standard deviation). For this reason, a corresponding smaller HP can cover a smaller part of the heat demands. Heat losses also increase due to the larger size and more intensive utilization of the storage tank. This effect increases with more idealization of the standby control (3).

The integrated coefficient of performance $C O P_{\beta}$ also takes the avoided cooling effort into account. Since the heat source fluctuates also more strongly with increasing standby control, this value decreases from the stationary design value 5.9 over 5.54 (scenario 1) and 5.52 (scenario 2) to 5.14 (scenario 3). 


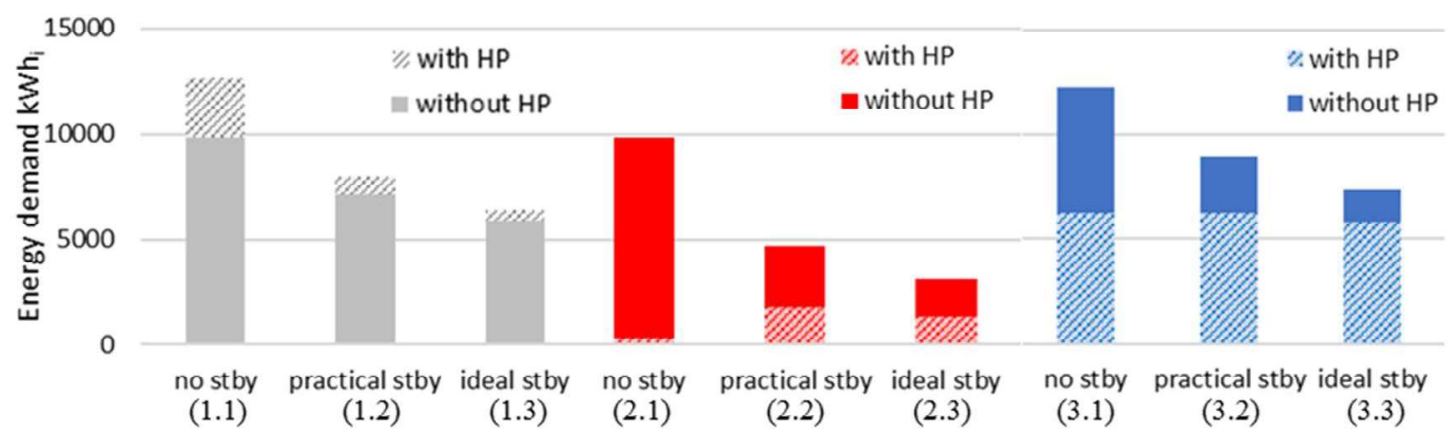

Figure 11. Remaining demand of useful energy forms: El. Energy (grey), Heating (red) and Cooling (blue) in different efficiency scenarios (1-3).

The remaining energy demand for the implementing of standby strategies and HPS divided according to the forms of useful energy is given in Figure 11: electricity (1), heating (2), cooling (3). The remaining demands with and without HP are displayed within a bar for the three scenarios. Figure 11 (1.1) shows that the electrical demand without standby control increases due to the use of HPs by $22 \%$. However, the use of standby control in combination with HPS reduces this demand by $37 \%$ (1.1. to 1.2) or $49 \%$ (1.1. to 1.3). The mean heat demand for a SROP of one week is reduced from $9,870 \mathrm{kWh}$ by $53 \%$ to $4,687 \mathrm{kWh}(2.1$ to 2.2$)$ through the sole practical standby strategy. Another $33 \%$ of heat demand can be saved by the ideal standby strategy (2.2. to 2.3). The HP without any standby strategy achieves the highest saving potential in the initial scenario (2.1). Since, with a few exceptions for the ideal standby, there is always sufficient heat available from the cooling process, the coverage of the cooling effort by the scenarios with HP changes only insignificantly $(3.1-3.3)$.

The results show that the HP works most efficiently for continuous demands without standbystrategy. Following the onion-layer approach for energy efficiency, the standby-strategies should be implemented first. In particular, this reduces the electrical power demand and the heating and cooling demand in equal measure. The installation of a standby manager is cost-effective compared to an HP. In addition, oversizing during retrofitting is prevented. This procedure can also reduce the HP capacity to be installed and thus the investment costs. The practical standby control provides the best compromise between reducing the electrical energy demand of the machines and efficiently covering the heating and cooling demands by HPs. The integration of HPS thus provides a system efficient solution, in which a rigorous ideal standby operation can be avoided, because it does not even significantly increase system energy efficiency any more. Anyway, an ideal standby operation is currently not state of the art and problematic, since a thermally stable condition is decisive for the processing accuracy of machine tools [31].

The representative case study in this paper includes numerous uncertainty factors. The full factorial experimental design ensures that the designed storages and the HP cover the entire range of thermal demand profiles. Despite this conservative design, which is intended for $100 \%$ supply coverage, a theoretically large number of storage units (twice as many process streams) can be reduced to a relatively small heat sink and a very small heat source storage. This reduction in the required number and size of the storages also underlines the case study results of Stoltze's combinatorial method [15]. 


\section{Conclusion and Outlook}

A main barrier to higher system efficiency in industry are high electrical, heating and cooling energy demands in non-value-adding production processes. This paper proposes the implementation of both an intelligent standby control and a HPS to simultaneously minimize energy demands and maximize waste heat recovery. In the case study, compared to the initial state, the electrical energy demand of the machines can be reduced by $27 \%$ and both the heating ( $83 \%$ ) and cooling ( $48 \%$ ) demand can be efficiently covered by HPs. Also, by implementing a HP, the cooling system becomes independent of the ambient temperature. This ensures the cooling of the machines at any time and the auxiliary chillers become obsolete. It also results in lower investment costs and space demand for the machine tool.

The HPs are dimensioned according to the minimum average heating demand, which enables high number of constant running hours. This allows the HP to operate in efficient operating conditions and keep the storage size and investment small while meeting heat demand reliably and at a high rate. Since conventional HP technology can be used to achieve the target temperatures in the application field, investment costs are reduced even further. In addition, the results show that an ideal standby manager requires a very high development effort, which struggles to obtain sufficient energy saving between practical and ideal standby managers. The ideal standby operation has no significant savings over the practical standby control system in combination with HPS. However, the paper shows different paths on the way to a high system efficiency.

In addition to the general main findings of this paper, the modular structure of the discrete event simulation environment developed in Plant Simulation provides comprehensive transferability to many conceivable case studies. This applies to the planning of new material flow systems as well as to the retrofit of existing ones. Material flow systems of any type, construction and size and their heating and cooling demands can be simulated. Also, any of the influencing factors described in chapter 3.1 can be considered. In the more comprehensive consideration of all influencing factors lies also the further research need.

Future work should investigate the extent material flow optimization approaches can consider, for example, production parts buffer size and spare parts provisioning [21], can be combined with the optimized planning of standby control and HPS. A faster delivery of spare parts, for example, ensures a more continuous material flow, which would be advantageous for HP operation. In contrast, large machining part buffers tend to lead to more discontinuous operation, making stricter standby operation more attractive and limiting HPS integration. In this context of planning new material flow systems, it is necessary to make an uncertain estimate of future and currently not yet measurable cooling and heating demands. On the one hand, there is a need for research into the most reliable model-based estimation possible of these values for the various operating states of the machines. On the other hand, a MC method should be applied with a growing number of uncertainty factors and a broad range of values. How the MC outputs can be interpreted and used for system efficient HPS dimensioning is part of future research. 


\section{Nomenclature}

A Machine availability

$\beta \quad$ Heat pump coverage rate

CC Composite curve

CIP Cleaning in Place

COP Coefficient of performance

$\mathrm{COP}_{\beta}$ Integrated coefficient of performance

DoE Design of Experiment

FTVM Fixed temperature, variable mass

$\dot{H} \quad$ Minimal average load

HEX Heat exchanger

HP Heat pump

HR Heat recovery

HRL Heat recovery loop

ISSP Indirect source and sink profile

MC Monte Carlo

MHR Maximum heat recovery

MN Multi-node

MTBF Mean time to failure

MTTR Mean time to repair

$\dot{Q}_{\text {i }} \quad$ Thermal load

SROP Stream-wise repeat operation period

ST: $\quad$ Stratified tank

TAM Time average model

$\mathrm{T}_{\text {cold }} \quad$ Temperature of the cold-water reservoir of a stratified tank

TES Thermal energy storage

$T_{\text {hot: }}$ Temperature of the hot-water reservoir of a stratified tank

TSM Time slice model

VLH Variable layer height

Acknowledments: The authors gratefully acknowledge the support of the EU project "Sustainable Process Integration Laboratory - SPIL", project No. CZ.02.1.01/0.0/0.0/15 003/0000456 funded by EU "CZ Operational Programme Research and Development, Education", Priority 1: Strengthening capacity for quality research.

\section{References}

[1] European Commission. Energy roadmap 2050. Luxembourg: Publications Office of the European Union; 2012.

[2] BMWi. Zahlen und Fakten Energiedaten: Nationale und Internationale Entwicklung. Berlin; 2018.

[3] Hoffmann C, Gerhardt N, Sandau F, Zimmermann B, Pape C, Bofinger S. Geschäftsmodell Energiewende: Eine Antwort auf das »Die-Kosten-der-Energiewende«-Argument. 2nd ed. Fraunhofer-Institut für Windenergie und Energiesystemtechnik, IWES, Kassel; 2015.

[4] Kemp IC. Pinch analysis and process integration a user guide on process integration for the efficient use of energy. 2nd ed. Oxford: Butterworth-Heinemann; 2007.

[5] Townsend DW, Linnhoff B. Heat and power networks in process design. // Heat and power networks in process design. Part I: Criteria for placement of heat engines and heat pumps in process networks. AIChE J. 1983;29(5):742-8.

[6] Klemeš JJ. Handbook of Process Integration: Minimisation of energy and water use, waste and emissions. Cambridge, UK: Woodhead Publishing; 2013.

[7] Wellig B, Grüninger A. Integration von Wärmepumpen in industrielle Prozesse mit Hilfe der Pinch-Analyse. News aus der Wärmepumpenforschung 2014;20:1-12.

[8] Preuß A. Einsatz einer Wärmepumpe in einem metallverarbeitenden Betrieb zur Nutzung technologischer Wärme. In: Bohne D, editor. Tagungsband 3. Energietechnisches Symposium Innovative Lösungen beim Einsatz 
Erneuerbarer Energien in Nichtwohngebäuden: 03. März 2011, Zwickau, 1st ed. Stuttgart: Steinbeis-Ed; 2011, p. $1-8$.

[9] Hennecke GmbH. Wärmerückgewinnung senkt Energieaufwand und Schadstoffemission: Presseinformationen. Neustadt/Wied; 2013.

[10] Hlavica M. Hartverchromung 21 -Prozesstechnik mit Vorbildcharakter: Die Metallveredelung Thoma in Heimertingen erhält Umweltpreis KUMAS Leitprojekte 2009. Galvanotechnik Sonderdruck (2010) 2009;4.

[11] Junge F, Helfert M, Abele E, Vogel F. Increase in energy efficiency of industrial production processes through thermal crosslinking of cutting-machine tools and cleaning machines by heat-pump technology. 12th IEA Heat Pump Conference 2017, Rotterdam; 2017.

[12] Watanabe C. Pioneering Industrial Heat Pump Technology in Japan. "Research and Development of Heat Pump and Thermal Storage Technologies in Industrial and Residential Sectors". Hanoi, Vietnam; 2013.

[13] Abele E, Panten N, Menz B. Data Collection for Energy Monitoring Purposes and Energy Control of Production Machines. Procedia CIRP 2015;29:299-304.

[14] Goy S. Stand-by-Betrieb von Maschinen und Anlagen: Entwicklung eines Stand-by-Managers zur energieeffizienten Produktionssteuerung. [Place of publication not identified]: Kassel University Press GmbH; Kassel University Press; 2016.

[15] Stoltze S, Mikkelsen J, Lorentzen B, Peterson PM, Qvale B. Waste-Heat Recovery in Batch Processs Using Heat Storage. Journal of Energy Resources Technology 1995;117(2):142-9.

[16] Seevers J-P, Schlosser F, Peesel R-H, Hesselbach J. Dimensioning of Heat Pump Systems Based on Pinch Analysis and Energy Monitoring Data.

[17] Junge F, Abele E, Vogel F. Qualification of Aqueous Part Cleaning Machines for the Use of Waste Heat in Industrial Production Companies. Procedia CIRP 2017;61:570-5.

[18] Bornemann T. Industrial waste heat utilization [Dissertation]: Kassel University Press GmbH.

[19] Denkena B, Helmecke P, Hülsemeyer L. Energy Efficient Machining with Optimized Coolant Lubrication Flow Rates. Procedia CIRP 2014;24:25-31.

[20] Augenstein E, Nelles J, Wurm A. Energieeffiziente Kühlsysteme für Werkzeugmaschinen: Konzept für eine bedarfsorientierte Kühlwasserversorgung. Werkstatttechnik online 2012;102(5).

[21] Kiesmüller GP, Zimmermann J. The influence of spare parts provisioning on buffer size in a production system. IISE Transactions 2018;50(5):367-80.

[22] Gutowski T, Murphy C, Allen D, Bauer D, Bras B, Piwonka T et al. Environmentally benign manufacturing: Observations from Japan, Europe and the United States. Journal of Cleaner Production 2005;13(1):1-17.

[23] Becker H. Methodology and Thermo-Economic Optimization for Integration of Industrial Heat Pumps [Thesis]. Lausanne: École Polytechnique Fédérale de Lausanne; 2012.

[24] Becker H, Maréchal F. Targeting industrial heat pump integration in multi-period problems. In: 11th international symposium on process systems engineering - PSE2012. Oxford: Elsevier; 2012, p. 415-419.

[25] Wallerand AS, Kermani M, Kantor I, Maréchal F. Optimal heat pump integration in industrial processes. Applied Energy 2018;219:68-92.

[26] Stampfli JA, Atkins MJ, Olsen DG, Wellig, Beat, Walmsley, Michael R. W., Neale JR. Industrial Heat Pump Integration in Non-Continuous Processes Using Thermal Energy Storages as Utility - A Graphical Approach Chemical Engineering Transactions 2018;70:901-6.

[27] Wang YP, Smith R. Time pinch analysis. Chemical Engineering Research and Design 1995;8(73):905-14.

[28] Schlosser F, Peesel R-H, Meschede H, Philipp M., Walmsley TG. Evaluation of a Stratified Tank based Heat Recovery Loop via Dynamic Simulation. Chemical Engineering Transactions 2018;70:403-8.

[29] Becker H. Methodology and Thermo-Economic Optimization for Integration of Industrial Heat Pumps [Dissertation]. Lausanne: École Polytechnique Fédérale de Lausanne; 2012.

[30] VDI. VDI-Wärmeatlas. 11th ed. Berlin, Heidelberg: Springer-Verlag GmbH; 2013.

[31] Mayr J, Jedrzejewski J, Uhlmann E, Alkan Donmez M, Knapp W, Härtig F et al. Thermal issues in machine tools. CIRP Annals 2012;61(2):771-91. 